\title{
Regresando a la división: el inverso del método del Campesino Ruso, enfoque recursivo
}

\author{
M.A. Murray-Lasso \\ Unidad de Enseñanza Auxiliada por Computadora \\ Departamento de Ingeniería de Sistemas. División de Estudios de Posgrado \\ Facultad de Ingeniería, UNAM
}

(recibido: abril de 1999; aceptado: diciembre de 2000)

\begin{abstract}
Resumen
La enseñanza de la recursión difícilmente se logra exhibiendo versiones recursivas de funciones como factorial y potencias como pretenden algunos libros de texto. Es necesario introducirse en más detalles procurando que los ejemplos no sean tan complejos, ya que complican más la enseñanza que el propio tema de la recursión. Las operaciones aritméticas elementales (suma, resta, multiplicación y división) proporcionan ejemplos de procesos recursivos ampliamente conocidos por los estudiantes, por lo tanto, son buenos candidatos para ilustrar recursiones no triviales. Este artículo trata en detalle la división de números enteros positivos partiendo de una cuenta de restas reiteradas (que para hacerla eficiente en caso de divisores grandes hay que restarle reiteradamente al dividendo, no solamente el divisor, sino diversos múltiplos del divisor estratégicamente escogidos). Se inicia por exhibir el método del Campesino Ruso (que coincide con un antiguo método egipcio) para multiplicar dos números. La inversión de dicho proceso, produce un método de división que aparentemente no ha sido discutido en la literatura. Se analiza la conexión tanto del método del Campesino Ruso como de su inverso, con el sistema binario de numeración. Al generalizar este método a otras bases, queda en evidencia que se trata del método que se enseña en la escuela primaria. Se hacen generalizaciones adicionales y se analizan como procedimientos recursivos, los cuales se programan en el lenguaje Logo. La conexión que se logra entre los temas descritos iluminará a los profesores de matemáticas tanto para el proceso de recursión, como para la verdadera esencia de la operación de división.
\end{abstract}

Descriptores: recursión, división, Logo, aritmética, Campesino Ruso

\section{Abstract}

The teaching of recursion can hardly be accomplished by showing recursive versions of the factorial and power functions as attempted by some textbooks. It is necessary to go into further detail with examples selected so that recursion and not the example itself becomes the difficult part of the process. Elementary arithmetic operations such as addition, subtraction, multiplication and division are processes which are well known to students and provide non-trivial examples of recursive processes, and are therefore good examples to use in teaching the topic. In the article the process of division of whole positive numbers is analyzed in detail starting from the fact that division is the counting of repeated subtractions which may be inefficient if the divisor is large and could be made more efficient if we subtract carefully chosen multiples of the divisor. The article starts by disclosing the Russian Peasant Method of multiplication (which coincides with an ancient Egyptian method). The inversion of this process becomes a method for division that has apparently not been discussed before in the literature. The connection of both the Russian Peasant Method and its inverse with the binary system of numbers is analyzed. When this method is generalized to other number bases it becomes evident that it is a particular case of the method ordinarily taught in elementary school. Further generalizations are introduced and analyzed as recursive processes that are programmed in Logo language. The connections made between the topics 
DOI: http://dx.doi.org/10.22201/fi.25940732e.2001.02n1.003

referred to will provide illumination to mathematics teachers not only in the process of recursion but also in the division process itself.

Keywords: recursion, division, Logo, arithmetic, Russian Peasant Method

\section{Introducción}

El profesor interesado en el uso de las computadoras como apoyo en el proceso enseñanza-aprendizaje, siempre busca métodos adicionales para ilustrar conceptos importantes en áreas como matemáticas y ciencias de la computación. La recursión es uno de los tópicos en ciencias de la computación en el cual los estudiantes tienen mayores proble. mas, por lo que se han buscado formas adicionales para enseñarla. Algunos libros introductorios de computación muestran la recursión, exhibiendo y analizando la versión recursiva de la función factorial o la función de elevar a una potencia (Levine, 1993). Esto no es suficiente para un tema tan rico con tantas facetas. Se ha concluido que las cuatro operaciones elementales de la aritmética se prestan a un tratamiento recursivo muy revelador. Los matemáticos que se interesan en los fundamentos del análisis aseguran que todas las funciones computables se pueden calcular utilizando la recursión (Landau, 1951 y Hermes, 1965).

Al estudiar el enfoque recursivo de la multiplicación surge uno de los esquemas alternativos para multiplicar dos números, llamado el método del Campesino Ruso (Bowers, 1961; Ore, 1976 y Menninger, 1992) que también fue utilizado en la antigüedad por los egipcios (Lara, 1983; Ore, 1976 y Menninger, 1992) y que está íntimamente relacionado con la representación binaria de los números. El método se puede generalizar a otras bases numéricas y su generalización a la base decimal nos lleva a los esquemas de multiplicación que se enseñan en la escuela primaria (Murray, 1995). Al estudiar el enfoque recursivo de la división, se puede dividir utilizando el inverso del método del Campesino Ruso de multiplicación y la generalización de este método al sistema decimal también nos lleva al esquema que se enseña ordinariamente en la escuela para dividir. El método del Campesino Ruso funciona para multiplicar en varios libros de aritmética y de matemáticas recreativas; sin embargo, no se ha encontrado su inverso en ninguno de ellos. Por lo anterior, se considera que sería de interés mostrar este sencillo método y cómo se presta a un enfoque recursivo, así como su generalización que lleva al método ordinario de división en notación decimal. Para ilustrar la recursión se utilizará el lenguaje LogoWriter para la PC porque se trata de un lenguaje educativo popular entre los docentes interesados en el cómputo educativo y porque, siendo un derivado de LISP, un lenguaje cuyo mecanismo de control de elección es la recursión, se presta muy bien para ilustrarla.

\section{Enfoque recursivo de la división}

La operación de división es la inversa de la multiplicación. Si el primer factor es F1 y el segundo F2, el resultado del producto es el resultado de sumar F2 un número de veces igual a $\mathrm{F} 1$, o lo que es lo mismo, sumar $\mathrm{F} 1$ un número de veces igual a F2. Dividir, por otro lado, significa encontrar cuántas veces cabe el divisor en el dividendo y cuántas veces podemos restarle el divisor al dividendo manteniendo el resultado no negativo (positivo o cero). Basados en esta idea, se puede escribir un procedimiento recursivo en Logo para implantar la división por restas sucesivas como sigue:

\section{PARA DIVISION :N :D \\ $\mathrm{SI}: \mathrm{N}<: \mathrm{D}[\mathrm{RE} 0]$ \\ RE $1+$ DIVISION :N - :D :D FIN}

donde $\mathrm{N}$ es el numerador o dividendo y $\mathrm{D}$ el denominador o divisor.

Este procedimiento recursivo es práctico cuando el resul. tado de la división, o sea, el cociente, es relativamente menor, ya que el procedimiento requiere un número pequeño de restas. Cuando el cociente es grande, el número de restas lo es también y el procedimiento se torna ineficiente tanto en tiempo, como en uso de memoria. Por esta razón conviene buscar mejores maneras de hacer la división.

\section{El método del Campesino Ruso para multiplicar}

En lugar de introducir una notación compleja con índices para explicar el método del Campesino Ruso para multiplicar, mejor se utilizará un ejemplo ilustrativo. Supóngase que se quiere multiplicar 42 por 79. Se colocan los factores como cabezas de dos columnas. Para obtener los siguientes números en la primera columna, se saca la mitad al número del renglón anterior, ignorando los restos cuando los haya, hasta que se llegue a la unidad como se muestra en la figura 1 .

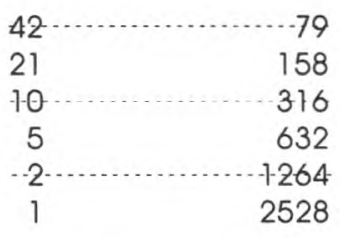

Figura 1 
Para obtener los números sucesivos de la segunda columna (encabezada por el segundo factor), duplíquese el número del renglón anterior. El procedimiento termina al llegar al renglón que corresponde al 1 de la primera columna, como se muestra en la figura 1 . Ahora táchense los renglones que tienen un número par en la primera columna. Las filas que se deben tachar en la figura 1 son las que corresponden a los pares de números: [42,79]; [10,316]; y [2,1264]. El resultado de la multiplicación se obtiene sumando los números no tachados en la columna de la derecha en la figura 1 . Este resultado es:

$$
158+632+2528=3318
$$

El resultado se puede verificar utilizando métodos convencionales de multiplicación.

\section{Conexión entre el método de multiplicación del Campesino Ruso y el sistema binario de numeración}

Para convertir un número en el sistema decimal al sistema de numeración binaria se puede utilizar el siguiente proceso:

Se toma el número y se divide entre dos; después se toma nota del cociente y del resto. El cociente de la operación anterior se divide entre 2 y se anota el cociente y el resto. Se procede de la misma manera hasta que uno de los cocientes sea cero. Posteriormente, se toman los restos y se yuxtaponen en orden inverso al que aparecieron, formando un número cuyos dígitos son ceros y unos. Este número es la representación binaria del número decimal del que se partió (Conte y Boor, 1980).

$\mathrm{Si}$ este proceso se le aplica al número 42 se obtiene la siguiente secuencia de cocientes y restos: (se incluye el número original en la lista de cocientes)

\section{Cocientes: 422110521}

Restos: $\quad 0 \quad 1 \quad 0 \quad 1001$

Al yuxtaponer los restos en orden opuesto al que se obtuvo se tiene el número binario 101010 el cual representa lo siguiente:

$$
\begin{aligned}
& 1 \times 2^{5}+0 \times 2^{4}+1 \times 2^{3}+0 \times 2^{2}+1 \times 2^{1}+0 \times 2^{0} \\
= & 32+0+8+0+2+0 \\
= & 42
\end{aligned}
$$

Ahora se escribe la multiplicación 42 × 79 de la siguiente manera:

$$
\begin{aligned}
42 \times 79 & =\left(1 \times 2^{5}+1 \times 2^{3}+1 \times 2^{1}\right) \times 79=(32+8+2) \times 79 \\
& =2528+632+158=3318
\end{aligned}
$$

Notamos que en el método del Campesino Ruso para multiplicar, los números de la columna derecha que fueron sumados para obtener el resultado (aquellos que no fueron tachados) son precisamente 2528,632 y 158 . También quedó demostrado que los cocientes sucesivos son precisamente los números de la primera columna de la figura 1 . De esa forma es muy sencillo predecir el resto en una división entre 2: si el dividendo es impar el resto es 1 , mientras que si es par, es 0 . La razón por la cual se tacharon ciertas filas es que corresponden a los términos que tienen multiplicador cero, ya que el resto correspondiente es nulo, pues el dividendo es par. Los números que no fueron tachados corresponden a 79, multiplicado por las potencias de 2 que tienen resto 1 pues el número dividido es impar. Por la manera en que se calcula la segunda columna de la figura 1 , lo que se obtiene al final es el producto de 42 (expresado como una suma de potencias de 2) por 79 .

\section{El inverso del método del Campesino Ruso para la división}

Supóngase que queremos dividir 3318 entre 42 . El método inverso del Campesino Ruso consiste en lo siguiente:

Formar dos columnas de números encabezados por 1 y el divisor, como se muestra en la figura 2.

$\begin{array}{rr}1 & 42 \\ 2 & 84 \\ 4 & 168 \\ 8 & 336 \\ -16 & \\ 32 & 672 \\ 64 & 2688\end{array}$

Figura 2

Los números en las filas debajo del encabezado se obtienen duplicando el número de la fila de encima como se muestra en la figura 2. Los cálculos se continúan hasta que el siguiente número que se escribe en la segunda co. lumna exceda al numerador o dividendo (para el ejemplo 3318). A continuación se construye un resto cambiante que se inicia restando del dividendo el núme. ro más grande de la columna derecha que no produce un resultado negativo. Por la manera en que se construye la tabla de la figura 2 el último número en la columna derecha siempre cumple esta condición. Después de la primera resta, el más grande número de la columna derecha que se le puede restar al resto cambiante sin 
resultado negativo es el que cumple la condición. Se tachan las filas que no cumplen la condición y se continúa el proceso hasta que el resto cambiante sea menor que el divisor que encabeza la columna derecha. Este último resto es el de toda la división. El cociente se obtiene sumando los números no tachados en la primera columna. Para el ejemplo, las restas sucesivas son: $3318-2688=630 ; 630-$ $336=294 ; 294-168=126 ; 126-84=42 ; 42-42=0$. Las restas exitosas corresponden a las filas no tachadas en la figura 2. De dicha figura el cociente es

$$
1+2+4+8+64=79
$$

con un resto de 0 , ya que ese fue el resultado de la última resta.

\section{El inverso del método del Campesino Ruso para la división como una recursión}

Escribamos procedimientos Logo recursivos para implantar el método inverso del Campesino Ruso de división para calcular N/D. Se hace través de dos procedimientos, más un tercero que los llama. En el primer procedimientollamado COLUMNAS calculamos las potencias sucesivas de 2 utilizando el parámetro $\mathrm{P}$ y sus productos con el divisor que no excedan al dividendo, utilizando el parámetro D. Para no tener que calcular estas cantidades de nuevo se almacenan en la LISTA que es la salida del procedimiento. Inicialmente LISTA está vacía y se construye con sublistas de dos elementos que corresponden a las dos columnas de la tabla de la figura 2 .

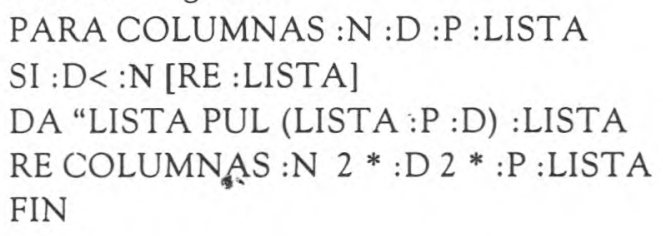

En un segundo procedimiento INVRUSO utilizamos la lista de salida de COLUMNAS para calcular el cociente con el método inverso del Campesino Ruso presentado en la sección anterior.

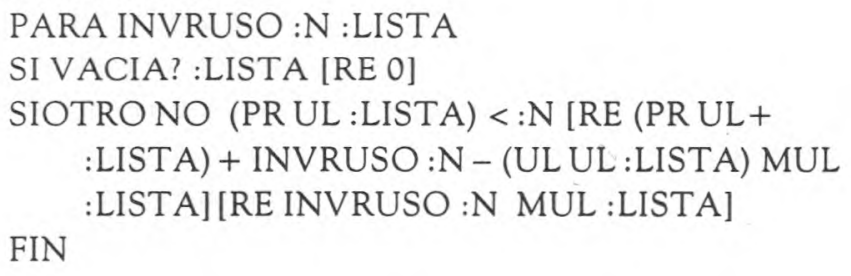

El tercer procedimiento, DIVISIONICR, simplemente llama a los otros dos procedimientos y da como salida el cociente N/D.

PARA DIVISIONICR :N :D
RE INVRUSO :N (COLUMNAS :N :D 1 []) FIN

Si se teclea ES DIVISIONICR 331842 en el centro de mandos, se obtiene la respuesta 79 en el área de trabajo de LogoWriter.

\section{Generalización del método inverso del Campe. sino Ruso para la división}

Si en lugar del sistema de numeración binario se utilizan otros sistemas de numeración se puede generalizar el inverso del método del Campesino Ruso para dividir. Tomemos como ejemplo el sistema ternario (base 3). El primer paso es construir dos columnas encabezadas por los números 1 y el divisor, triplicando los números en ambas columnas para cada fila hasta que el siguiente número en la segunda columna excede al dividendo, como se muestra en la figura 3.

$\begin{array}{rrr}1 & 42 & 1 \\ 3 & 126 & 2 \\ 9 & 378 & 2 \\ 27 & 1134 & 2\end{array}$

Figura 3

Ahora se calcula un resto cambiante, excepto que en vez de una sola resta para cada número se intenta restar cada número de la columna 2 cuantas veces se pueda sin que se obtenga un resultado negativo. Los intentos se hacen de mayor a menor entre los números de la columna 2. Por la manera como se construyó la tabla siempre se puede restar el último número de la columna 2 por lo menos una vez. Se escrie en una tercera columna el número de veces que se puede realizar cada resta. Para un sistema ternario ningún número en la tercera columna debe exceder 2. Las restas sucesivas son: $3318-1134=2184$; $2184-1134=1050 ; 1050-378=672 ; 672-378=294 ; 294$ $-126=168 ; 168-26=42 ; 42-42=0$. El número de restas de cada número diferente en la segunda columna se muestra en la tercera columna de la figura 3. El último resultado de las restas es el resto de toda la división. En este caso resulta 0 .

El cociente se calcula multiplicando los números en la tercera columna por los números en la primera columna y sumando. En nuestro caso el cociente es: $2 \times 27+2 \times 9+$ $2 \times 3+1 \times 1=54+18+6+1=79$ como en el método original.

El lector deberá notar que el método inverso del Campesino Ruso está basado en la fórmula

$$
\text { dividendo }=\text { cociente } \mathrm{x} \text { divisor }+ \text { resto }
$$

donde el cociente queda expresado como la suma de productos de unos coeficientes por potencias de la base 
numérica que se escogió. Para expresar adecuadamente el cociente en el sistema numérico de base $b$, los coeficientes deben ser todos menores que la base. En el caso de la base 2 , los coeficientes deben ser 0 ó 1 ; ésa es la razón por la cual basta tachar los números que corresponden a 0 . En un sistema ternario, en contraste, los coeficientes pueden ser 0,1 ó 2 . Encontrar los coeficientes en la expresión del dividendo es muy similar a tener un conjunto de objetos y contarlos en el sistema de base b. Lo que se hace es tratar de ver cuál es el tamaño del mayor conjunto que se puede formar con $\mathrm{b}$ a alguna potencia. Por ejemplo, si la base es 10 y el número de objetos es más grande que cien y menor que mil, se formarían tantos conjuntos de cien como se pudiera. Los objetos restantes que no completaran el último ciento, se dividirían en tantos conjuntos de diez objetos como se pudiera y posiblemente quedarían algunos objetos fuera. Estos últimos quedarían tal cual como unidades. Si en vez de base 10 se utiliza la base $3,1 a$ primera cosa a determinar sería cuál es la potencia de 3 con la que se formarían los conjuntos más grandes. Podrían formarse 1 ó 2 de estos conjuntos. Los restantes se agruparían en conjuntos de 3 elevado a una potencia igual a la de los mayores menos uno. Trabajando de igual forma eventualmente se llega a las unidades. El método garantiza expresar el número total en el sistema numérico de base $b$, porque necesariamente todos los coeficientes estarán entre 0 y b-1, ya que si en algún caso apareciera un número mayor o igual que b esto significaría que en el paso anterior se hubiera podido formar por lo menos un conjunto adicional.

La división decimal como un caso particular del método generalizado inverso del Campesino Ruso para dividir

Se utilizará el sistema de numeración decimal o de base 10 , para dividir 3328 entre 42, utilizando la generalización del método inverso del Campesino Ruso formaríamos la tabla de la figura 4 .

$\begin{array}{rr}1 & 42 \\ 10 & 420\end{array}$

Figura 4

Se debe averiguar cuántas veces podemos restar 420 de 3328 y deduciríamos 7 con resto 388 . Luego se encontrará que se puede restar 42 hasta 9 veces dejando un resto de 10 . De lo anterior se deduce que el cociente es $7 \times 10+9 \times 1=79$. Ahora, si se comparalo hechoconel proceso normal de división que se enseña en la escuela, determinar el número de veces que se puede restar 420 de 3328 es lo mismo que dividir 3328 entre 420 (que es lo mismo que dividir 332.8 entre 42 ). Esto nos da 7, la primera cifra del cociente. Se encuentra el resto al hacer la resta de 3328 el producto de 7 por 420 . Esto nos da como resultado 388. Luego, encontrar cuántas veces se puede restar 42 de 338 es lo mismo que encontrar el cociente de 388 entre 42 . Obtenemos como respuesta 9 . Se calcula el resto restando de 388 el producto de $9 \times 42$, lo que deja un resto de 10 (Fig. 5)

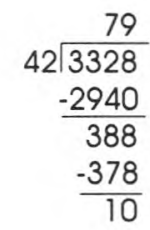

Figura 5

De la figura 5 vemos que el proceso de "bajar el 8" después de la determinación de la primera cifra del cociente y el primer resto, es una consecuencia del hecho que el número que se resta para determinar el primer resto termina en ceros y por lo tanto, no afecta los últimos dígitos a la derecha. Esta circunstancia se parece a la que se presenta en la multiplicación, en la cual se corren a la izquierda los resultados de los productos del multiplicando por las subsecuentes cifras del multiplicador; el corrimiento es equivalente a multiplicar las cantidades por 10 , o lo que es lo mismo, añadir un cero a la derecha.

Debería ser evidente que el proceso ordinario de división es una generalización del método inverso del Campesino Ruso para dividir, el cual se obtiene invirtiendo el método del Campesino Ruso para multiplicar. Si creemos en la teoría construccionista del aprendizaje (Papert, 1993), el conectar estas ideas a lo que ya sabemos del proceso de división enriquece y consolida nuestro conocimiento de toda la aritmética. En la siguiente sección conectamos este conocimiento con el proceso de recursión.

Procedimiento recursivo en Logo para la división ordinaria en el sistema decimal

A continuación se muestra un procedimiento en Logo para hacer la división basada en las ideas anteriores. Lo primero que se necesita en el procedimiento de división que corresponde a las figuras 4 y 5 es conocer la potencia 
de 10 por la que debe multiplicarse el divisor en el último número en la columna derecha de la figura 4. Llamaremos a este número EXPONENTE. Se calculará en el procedimiento DECDIVIDE el cual llama al procedimiento recursivo DIVISION2 y le será pasado como un parámetro. Para determinar el valor de EXPONENTE se utiliza el hecho simple de que un cálculo aproximado, el cual posiblemente excede en a lo más 1 el valor correcto, se logra tomando la diferencia de número de cifras entre el dividendo y el divisor expresados en notación decimal. No es importante si nos excedemos en 1 al calcular el valor de EXPONENTE puesto que el precio que se paga es un paso más en el proceso, produciéndose un cero, que al agregarlo al resultado final no lo afecta. Una vez que se determina el valor de EXPONENTE en DIVISION2 se multiplica el divisor por 10 elevado a EXPONENTE y se utiliza esta cantidad como divisor para dividir al dividendo. El resultado de esta última operación debe tener un dígito, por lo que se puede obtener eficientemente utilizando el procedimiento DIVISION dado al principio del artículo. Al dígito, que en el programa se le llama $\mathrm{T}$, se le debe interpretar en el resultado final como la contribución al cociente del valor del dígito multiplicado por 10 elevado a EXPONENTE. El resto de la contribución al cociente, proviene de calcular el resto, quitando lo que corresponde a $\mathrm{T}$, y así calcular lo que sería el cociente para dicho resto utilizando el mismo divisor. Esta situación es perfecta para una autollamada al procedimiento DIVISION2 con los argumentos adecuados. Para hacer más claro el listado utilizamos las variables intermedias $T$ y $R$ para la contribución al cociente y el resto, respectivamente. Dado que cada dígito que aparece en el cociente reduce el exponente que se requiere en uno, la autollamada se hace con el tercer parámetro igual a EXPONENTE - 1. Para detener la recursión, cuando el resto (que se usa como dividendo en las siguientes llamadas al procedimiento DIVISION2) tiene la misma magnitud que el divisor (lo que sucede cuando EXPONENTE $=0$ ), entonces simplemente se saca como salida la de DIVISION.

\section{PARA DIVISION2 :N :D :EXPONENTE}

$S I:$ EXPONENTE $=0[$ RE DIVISION $: N: D]$

DA "T DIVISION :N :D * POTENCIA 10 :EXPONENTE

DA "R :N - :D* :T* POTENCIA 10 :EXPONENTE

RE $(: T *$ POTENCIA $10:$ EXPONENTE) + DIVISION2 $: R: D:$ EXPONENTE - 1

FIN

Este procedimiento recursivo utiliza al procedimiento DIVISION que se dio al principio del artículo y es llamado por el siguiente procedimiento:
PARA DECDIVIDE :N :D

DA “EXPONENTE (CUENTA :N) - (CUENTA :D)

RE DIVISION2 : $N$ :D :EXPONENTE

FIN

El procedimiento DIVISION2 requiere el cálculo de potencias de 10. LogoWriter no proporciona esta función, la cual es

\section{PARA POTENCIA :BASE :EXPONENTE SI : EXPONENTE $=0$ [RE 1] \\ $\mathrm{RE}: \mathrm{BASE}$ * POTENCIA :BASE :EXPONENTE -1 FIN}

Se remplazará la multiplicación de T por una potencia de 10 , por un procedimiento más primitivo AGCEROS que agrega ceros a la derecha de un número, generando el mismo resultado. La nueva versión de DIVISION2 con estos cambios es

\section{PARA DIVISION2 : $\mathrm{N}: \mathrm{D}:$ :EXPONENTE} $S I:$ EXPONENTE $=0[$ RE DIVISION $: N: D]$ DA “T DIVISION :N AGCEROS :EXPONENTE :D DA " $\mathrm{R}: \mathrm{N}-: \mathrm{D} *$ AGCEROS :EXPONENTE :T RE (AGCEROS :EXPONENTE $:$ T) + DIVISION2 :R :D :EXPONENTE-1

FIN

donde el procedimiento AGCEROS es

PARA AGCEROS :DIGITOS :NUMERO
DA " $X$ :NUMERO
REPITE :DIGITOS [DA "X (PALABRA :X "O)]
RE :X
FIN

Para probar los procedimientos, simplemente se teclea ES DECDIVIDE 332842 y debería aparecer 79 en el área de trabajo de LogoWriter.

\section{Generalización adicional del método de división}

Se ha procedido a deducir el método ordinario de división que se enseña en la escuela como una generalización del método inverso del Campesino Ruso. Todos los métodos que se han presentado expresan el cociente como un número en un sistema de numeración y utilizan la propiedad distributiva de la multiplicación. De hecho, el cociente no tiene que estar expresado en al. gún sistema de numeración específico (es decir, como una suma de coeficientes por potencias de la base de numeración seleccionada). Basta que se exprese el cociente 
como una suma y se aplique la propiedad distributiva de la multiplicación.

Al reconocer que la división es una cuenta de restas repetidas y el primer procedimiento recursivo DIVISION simplemente cuenta el número de veces que se podría restar el divisor del dividendo sin dar un resultado negativo, se nota que si el cociente es grande el número de restas sería grande y el procedimiento ineficiente. Por ejemplo, en la división $3328 / 42$ se tendrían que hacer 79 restas. Si se sabe que va a haber tantas restas, de una vez podríamos restar decenas de 42's hasta que ya no se puedan restar mas decenas. Después continuaría la resta de 42 's hasta que se obtenga un resultado inferior a 42 . Ese es el método decimal. Pero de igual modo podríamos haber restado cincuenta 42's, luego veinte 42's, luego cinco 42's y finalmente dos veces dos 42's. Lo que se necesita es tener un número adecuado de múltiplos de 42 de tal manera que se logre formar cualquier número que corresponda al cociente. Esto es similar al problema de tener una variedad adecuada de monedas o billetes para dar cambio en la operación de una tienda. Una posibilidad es tener unos, doses, cincos, dieces, veintes, cincuentas, cienes, doscientos, quinientos, miles, dosmiles, cincomiles, etc.

Se intentará de nuevo la división 3328/42. En la figura 6 se ha construido una tabla de multiplicadores y los mismos multiplicadores por el divisor 42 , parecida a las de las

$\begin{array}{rrr}1 & & 42 \\ 2 & X X & 84 \\ 5 & X & 210 \\ 10 & & 420 \\ 20 & X & 840 \\ 50 & X & 2100\end{array}$

figuras 3 y 4 con los números 1, 2, 5, 10, 20, 50 como se sugirió antes.

\section{Figura 6}

No incluimos 100 en la primera columna de la figura 6 porque en la segunda columna se hubiera excedido el dividendo 3328. Ahora comenzamos a restar de 3328 las diferentes cantidades en la columna derecha de la figura 6 para obtener $3328-2100=1228$. Se nota que no se puede restar el número que corresponde a 50 más que una vez, por lo que se escribe una $\mathrm{X}$ a la derecha de 50 . Ahora se resta 840 para obtener $1228-840=388$. Nuevamente es claro que no se puede restar otro 840 por lo que se marca una $X$ a la derecha de 20. Luego se resta 210 (ya que no se logró restar 420 sin obtener un resultado negativo) para obtener $388-210=178$ y como no se puede repetir la operación se pone una sola $\mathrm{X}$ a la derecha de 5 . Ahora se resta 84 para obtener $178-84=94$. Aquí sí se puede repetir la operación de modo que se obtiene $94-$ $84=10$, por lo que ponemos 2 X's a la derecha de 2 . De aquí en adelante no se continua con las restas pues el sobrante es menor que 42 , por lo que se finaliza el proceso con un resto igual a 10 . Finalmente, para obtener el cociente se suman los números de la columna izquierda multiplicados cada uno por el número de X's a su derecha. Se obtiene $50+20+5+2 \times 2=79$.

Podríamos escoger cualesquiera números en la primera columna de la figura 6 con los cuales pudiéramos formar el cociente como una suma. En el primer procedimiento de este artícuio se formó cualquier cociente sumando puros unos. Con el método inverso del Campesino Ruso escogimos potencias de dos. Luego se generalizó a potencias de 3 y 10 y pudieron haberse utilizado otras bases si se deseaba. Por último se escogerán números similares a las denominaciones de monedas y billetes. Se pudieron haber escogido otras cantidades. Los que se escogieron para la figura 6 tienen la ventaja de que realmente sólo es necesario hacer dos cálculos: multiplicar por 2 y por 5 (que se puede lograr agregando un cero y sacando mitad) y agregar los ceros necesarios para obtener los productos correspondientes a $20,50,200,500$, etc.

\section{Conclusiones}

Al enseñar recursión con base en la construcción de conocimientos nuevos sobre conocimientos previos es muy conveniente exhibir y dejar que los estudiantes resuelvan problemas que requieren recursion en áreas con las que están familiarizados. Una de dichas áreas es la de las operaciones aritméticas que se enseñan en la escuela primaria. En este artículo se tomó el proceso de división, el cual se puede ver como la cuenta de restas repetidas, que se aplica recursivamente al resto después de cada vuelta. En algunos casos, el algoritmo obtenido es ineficiente y se inicia la búsqueda de un algoritmo más eficiente. Encontramos que es más eficiente el algoritmo asociado al método inverso del Campesino Ruso, que se basa en la representación binaria del cociente de la multiplicación. Este método se generaliza a orras bases y cuando se usa la base decimal, el algoritmo coincide con el método estándar de división que se enseña en la primaria.

El proceso de división se ilustra mejor construyendo el cociente esencialmente con cualquier conjunto de multiplicadores que contengan la unidad. Este es el momento adecuado para decir algo sobre trucos espe. ciales para multiplicación por algunos números especiales. Por ejemplo, para multiplicar un número por $97 \mathrm{se}$ 
multiplica el número por 100 agregándole dos ceros a la derecha y luego se le resta el triple del número. Para multiplicar por 35 se puede multiplicar por 50 añadiendo dos ceros y sacando mitad y luego se le resta al resultado el número original con un cero añadido, finalmente se resta la mitad del anterior. $(35=50-10-5)$.

El método ordinario de división decimal también se puede enfocar recursivamente como los otros casos. El viaje completo, por hacerse sobre terreno en el que antes se ha pisado, ilumina tanto la aritmética como la recursión, proporcionando una rica experiencia de aprendizaje. La computadora se utiliza para materializar los conceptos y poner a prueba las ideas. Logo es un medio ideal para lo anterior. El hecho de que la división, que es la más difícil de las cuatro operaciones aritméticas elementales, se pueda hacer en forma sencilla simplemente sabiendo duplicar números (lo cual se puede hacer sumando), comparar, sumar y restar siguiendo reglas sencillas, generalmente intriga a los estudiantes, ya que pensaban que el proceso era más complejo. A los alumnos les interesa saber por qué funcionan los esquemas, y si siempre resultan. Si se presenta en clase una situación novedosa los estudiantes le exigen al maestro la demostración matemática de que el método funciona siempre. Normalmente, los estudiantes quedan satisfechos con aprender reglas sin saber por qué funcionan. Pero ante la aparente novedad se les despierta la curiosidad. Este enfoque consiste en regresar a algo que los estudiantes ya conocen y mostrar una diferente manera dehacerlo, posiblemente más sencilla y menos laboriosa, ello proporciona una bienvenida motivación que completa el ambiente propicio para que los estudiantes aprovechen experiencias ricas en aprendizaje, las cuales necesitan implementarse a las clases de matemáticas de cualquier nivel.

\section{Referencias}

Landau E. (1951). Foundations of Analysis: The Arithmetic of Whole, Rational, Irrational and Complex Numbers. Chelsea Publishing Company, Nueva York.

Hermes H. (1965). Enumerability, Decidability, Computability: An Introduction to the Theory of Recursive Functions. Academic Press, Inc., Publishers, 9-65.

Murray-Lasso M.A. (1995). La Recursion y su enseñanza via Logo y la multiplicación.

Memoria del XI Simposium Internacional de Computación en la Educación, Puebla, Pue., México, octubre, pp. 371 - 376.

Lara-Aparicio M. (1983). Antología de matemáticas, Vol. I, Capítulo 8, M. Willerding, Métodos Antiguos de Computación, Universidad Nacional Autónoma de México, México, DF, p. 61.

Conte S.D y Boor C. (1980). Elementary Numerical Analysis: An Algorithmic Approach, (Third Edition, International Student Edition), Mc Graw-Hill Kogakusha, Ltd., Tokyo.

Bowers H. y Bowers J.E. (1961). Arithmetical Excursions: An Enrichment of Elementary Mathematics, Dover Publications. Nueva York.

Levine G. (1993). Introducción a la computación y a la programación estructurada, McGraw-Hill Latinoamericana de México. México.

Ore O. (1976). Number Theory and Its History, Dover Publicactions, Inc., Nueva York, p. 38.

Menninger K. (1992). Number Words and Number Symbols: A Cultural History of Numbers, Dover Publications, Inc., Nueva York, p. 360.

Papert S. (1993). The Children's Machine: Rethinking School in the Age of the Computer, Basic Books, New York.

\section{Semblanza del autor}

Marco Antonio Murray-Lasso. Realizó la licenciatura en ingeniería mecánica-eléctrica en la Facultad de Ingeniería de la UNAM. El Instituto de Tecnología de Massachussetts (MIT) le otorgó los grados de maestro en ciencias en ingeniería eléctrica y doctor en ciencias cibernéticas. En México, ha laborado como investigador en el Instituto de Ingenería y como profesor en la Facultad de Ingeniería (UNAM) durante 36 años; en el extranjero, ha sido asesor de la NASA en diseño de circuitos por computadora para aplicaciones espaciales, investigador en los Laboratorios Bell, así como profesor de la Universidad Case Western Reserve y Newak College of Engineering, en los Estados Unidos. Fue el presidente fundador de la Academia Nacional de Ingeniería de México; vicepresidente y presidente del Consejo de Academias de Ingeniería y Ciencias Tecnológicas (organización mundial con sede en Washington que agrupa las Academias Nacionales de Ingeniería). Actualmente, es jefe de la Unidad de Enseñanza Auxiliada por Computadora de la División de Estudios de Posgrado de la Facultad de Ingeniería de la UNAM, investigador nacional y consultor de la UNESCO. 